\title{
Isolation and partial characterization of cyclic lipopeptide antibiotics produced by Paenibacillus ehimensis B7
}

Zhaohui Huang ${ }^{1,2^{*}}, \mathrm{Yu} \mathrm{Hu} \mathrm{u}^{1,2}$, Linfei Shou ${ }^{3}$ and Mingxu Song ${ }^{1,2}$

\begin{abstract}
Background: The prevalence of drug-resistant bacteria has encouraged the search for novel antimicrobial compounds. Food-associated microorganisms, as a source of new antibiotics, have recently received considerable attention. The objective of this study was to find novel antimicrobial agents produced by food microorganisms.

Results: A bacterial strain B7, which has potent antimicrobial activity, was isolated from a sample of dairy waste. This strain was identified as Paenibacillus ehimensis based on the 16S rRNA gene sequence analysis, physiological and biochemical characterization. Two active compounds (PE1 and PE2) were obtained from P. ehimensis B7. Mass spectrometry (MS) analysis showed that the molecular masses of PE1 and PE2 were 1,114 and 1,100 Da, respectively. The tandem MS and amino acid analysis indicated that PE1 and PE2 were analogs of polypeptin, and PE2 was characterized as a new member of this family. Both compounds were active against all tested bacterial pathogens, including methicillin resistant Staphylococcus aureus, Escherichia coli, and pan-drug resistant Pseudomonas aeruginosa clinical isolate. Time-kill assays demonstrated that at $4 \times$ MIC (minimum inhibitory concentration), PE1 and PE2 rapidly reduced the number of viable cells by at least 3-orders of magnitude, indicating that they were bactericidal antibiotics.
\end{abstract}

Conclusions: In the present work, two cationic lipopeptide antibiotics (PE1 and PE2) were isolated from $P$. ehimensis B7 and characterized. These two peptides showed broad antimicrobial activity against all tested human pathogens and are worthy of further study.

Keywords: Paenibacillus ehimensis, Lipopeptide antibiotics, Drug-resistant bacteria

\section{Background}

Clinical infection due to drug-resistant bacteria is a serious challenge to patient safety [1,2]. In the United States, methicillin-resistant Staphylococcus aureus (MRSA) is estimated to cause $\sim 19,000$ deaths per year [3]. MRSA is also a considerable threat in China, where the resistance ratio among hospital-acquired infections reaches almost 90\% [4,5]. Apart from MRSA, several multidrug-resistant (MDR) and pan-drug-resistant (PDR) Gram-negative bacteria, including Pseudomonas aeruginosa, Escherichia coli, Acinetobacter baumannii, and Klebsiella pneumoniae, are

\footnotetext{
* Correspondence: hzhwxsy@126.com

${ }^{1}$ Clinical laboratory, the Fourth Affiliated Hospital of Soochow University, 200 Huihe Road, Wuxi 214062, China

${ }^{2}$ Clinical laboratory, the Fourth People's Hospital of Wuxi, 200 Huihe Road, Wuxi 214062, China

Full list of author information is available at the end of the article
}

emerging as additional major threat to public health [6,7]. Unfortunately, few novel drugs have been developed specifically for MDR/PDR Gram-negative bacteria in recent years [8-10]. The development of new antimicrobial agents cannot keep up with the evolution of bacterial resistance. Thus, more efforts should be placed on discovering and developing new antimicrobial agents.

As a source of new antibiotics, food-associated microorganisms have recently received increased attention. The well-known active compounds produced by these strains are peptide antibiotics, such as lantibiotics and lipopeptides [11-13]. Many of them are potentially useful in medical and food applications due to their low intestinal toxicity. To obtain antimicrobial agents that are novel safe and potent, a lot of food bacteria were isolated and screened for their antimicrobial activity. In this work, strain B7, a new bacterial isolate from a sample of dairy waste, was found to 
produce antibiotics against both Gram-positive and Gram-negative human pathogens. Based on the $16 \mathrm{~S}$ rRNA gene sequence analysis as well as physiological and biochemical characterization, strain B7 was identified as Paenibacillus ehimensis. After isolation and purification of the fermentation products, the chemical structure and biological characteristics of the active compounds produced by $P$. ehimensis $\mathrm{B} 7$ were determined.

\section{Methods}

\section{Strains and culture conditions}

Samples of dairy waste were collected from a local dairy industry in Wuxi. The dairy waste samples were suspended in $0.1 \%$ sterile peptone water and antibiotic producing strains were isolated using a competitive inhibition method as previously described [14]. Nutrition broth was used for routine culture. The active compounds were produced in synthetic Katznelson and Lochhead (KL) medium, which had the following composition (in $\mathrm{g} / \mathrm{L}$ ): glucose, 5; $\left(\mathrm{NH}_{4}\right)_{2} \mathrm{SO}_{4}, 1.5 ; \mathrm{MgSO}_{4} 7 \mathrm{H}_{2} \mathrm{O}, 0.2 ; \mathrm{NaCl}, 0.1 ; \mathrm{CaCl}_{2}, 0.1$; $\mathrm{FeSO}_{4} 7 \mathrm{H}_{2} \mathrm{O}, 0.01 ; \mathrm{ZnSO}_{4}, 0.01 ; \mathrm{MnSO}_{4} \mathrm{H}_{2} \mathrm{O}, 0.0075$; and $\mathrm{KH}_{2} \mathrm{PO}_{4}$ 2.7. The medium was autoclaved and brought to a $\mathrm{pH}$ of 7.2. Staphylococcus epidermis CMCC 26069 was purchased from the National Center for Medical Culture Collections. S. aureus ATCC 43300, S. aureus ATCC 25923, E. coli ATCC 35218, and P. aeruginosa ATCC 27853 were purchased from the American Type Culture Collection (ATCC). Clinical isolates (P. aeruginosa 5215 and $E$. coli 5539) were isolated from patients at the Fourth People's Hospital of Wuxi, Wuxi, China. The tested strains that were used to determine the sensitivity to the active compounds were routinely grown at $37^{\circ} \mathrm{C}$ on a nutrient agar or in a nutrient broth. For long-term storage, all of the strains were stored in $20 \%(\mathrm{v} / \mathrm{v})$ glycerol at $-80^{\circ} \mathrm{C}$. This study was approved by the Ethics Committee of the Fourth People's Hospital of Wuxi.

\section{Strain identification}

The morphology of strain B7 was examined by light microscopy after Gram-staining and spore staining. The physiological and biochemical characteristics of the isolate was assessed according to previously described methods [15]. Motility was determined using sulfide-indole-motility medium. Fatty acid methyl esters were extracted and analyzed by the Sherlock Microbial Identification system (MIDI, Newark, DE) according to the manufacturer's instructions. All assays were performed in triplicate. The $16 \mathrm{~S}$ rRNA gene of strain B7 was amplified by PCR with the universal primers $27 \mathrm{~F}$ and $1541 \mathrm{R}$ and sequenced [16]. Phylogenetic trees were constructed using the neighbor-joining and maximum-parsimony algorithm within MEGA4 [17]. The DNA-DNA hybridization between B7 and Paenibacillus ehimensis IFO $15659^{\mathrm{T}}$ was performed using the thermal denaturation method [14].

\section{Production and purification of active compounds}

Strain B7 maintained on nutrient agar slants was inoculated into $50 \mathrm{~mL}$ of nutrient broth and cultivated at $30^{\circ} \mathrm{C}$ for $24 \mathrm{~h}$. The seed culture of strain B7 was transferred to a $2 \mathrm{~L}$ Erlenmeyer flask that contained $500 \mathrm{~mL}$ of the KL medium. The culture was incubated on a rotary shaker $(200 \mathrm{rpm})$ at $30^{\circ} \mathrm{C}$ for $3 \mathrm{~d}$. After centrifugation at $4500 \mathrm{~g}$ for $30 \mathrm{~min}$ at $4^{\circ} \mathrm{C}$, the cell-free supernatant was loaded onto a column packed with Amberlite XAD-16 resin (Sigma, St. Louis, $\mathrm{MO})$. The column was washed with distilled water prior to elution with stepwise gradients of aqueous methanol (30, 60 , and $100 \%, v / v)$. Each fraction was concentrated and assessed for activity using the paper disc method. The active fraction was evaporated and dried before being redissolved in acetonitrile. The concentrated solution was then applied to a $\mathrm{C}_{18}$ SPE column (Hardwee, Germany). The column was washed with five bed volumes of distilled water, followed by five bed volumes of an acetonitrile/water mixture $(20: 80, \mathrm{v} / \mathrm{v})$. The fraction that contained the active compounds was eluted from the column by washing with three bed volumes of an acetonitrile/water mixture (68:32, v/v). Further purification was performed using a preparative HPLC system (Dalian Elite, Dalian, China) that was equipped with an YMC-pack DOS-A C18 $(5 \mu \mathrm{m}, 250 \times 20 \mathrm{~mm})$ column. The mobile phase consisted of Milli-Q water that contained $0.02 \%$ trifluoroacetic acid and acetonitrile. A linear gradient of $15 \%$ to $55 \%$ acetonitrile $(40 \mathrm{~min}$ ) was used for elution at a flow rate of $10 \mathrm{~mL} / \mathrm{min}$. UV detection was performed at a wavelength of $210 \mathrm{~nm}$. Fractions from multiple runs were collected and combined for the subsequent antimicrobial activity assays. The active fractions were passed through the HPLC column two consecutive times.

\section{Amino acid analysis}

Approximately $300 \mu \mathrm{g}$ of the purified compound in $0.4 \mathrm{ml}$ of $6 \mathrm{M} \mathrm{HCl}$ with $0.1 \%$ phenol was hydrolyzed at $110^{\circ} \mathrm{C}$ for $16 \mathrm{~h}$. Amino acid analyses was performed using ion-exchange chromatography with a Hitachi L-8900 amino acid analyzer (Tokyo, Japan) according to the method described by Qian et al. [18]. The absolute configuration of amino acids was determined using 1-fluoro-2,4-dinitrophenyl-5-D-leucinamide (D-FDLA) and 1-fluoro-2,4-1-fluoro-2,4-dinitrophenyl-5-L-leucinamide (L-FDLA) as derivatizing agents. FDLA derivative analysis was performed as previously described [19].

\section{Mass spectrometry analysis}

Electrospray ionization (ESI) mass spectra were acquired in positive ion mode on a Thermo Finnigan LCQ mass spectrometer (Thermo Electron Corporation, San Jose, CA, USA). The ESI-mass spectrometry (MS) conditions included a capillary voltage of $40 \mathrm{~V}$, a source voltage of $4.5 \mathrm{kV}$, and a capillary temperature of $300^{\circ} \mathrm{C}$. To obtain 
the amino acid sequences, collision induced dissociation (CID) was applied to the purified lipopeptide antibiotics.

\section{Antibacterial activity assay}

During fermentation and purification, antimicrobial activity was determined using the paper disc method [14]. The minimum inhibitory concentrations (MICs) of the purified antibiotics were determined using a microbroth dilution method according to the National Committee for Clinical Laboratory Standards (2009). The final concentrations of the antibiotics in the medium ranged from 1 to $64 \mu \mathrm{g} / \mathrm{mL}$. MICs were measured after incubation at $37^{\circ} \mathrm{C}$ for $20 \mathrm{~h}$. To determine the effect of divalent cations on the mode action of purified compounds, $10 \mathrm{mM} \mathrm{CaCl}_{2}$ or $\mathrm{MgCl}_{2}$ was added to the test medium.

\section{Time-kill assays}

To further evaluate the antimicrobial characteristics of the purified compounds, time-kill experiments were performed as previously described [18]. The active compound was added to a logarithmic-phase broth culture of approximately $10^{6} \mathrm{cfu} / \mathrm{mL}$ to yield concentrations of 0 and $4 \times$ MIC. The cultures were incubated with shaking $(120 \mathrm{rpm})$ at $37^{\circ} \mathrm{C}$ for $24 \mathrm{~h}$. Surviving bacteria were determined after $0,1,3,6$, and $24 \mathrm{~h}$ of incubation by subculturing $100 \mu \mathrm{L}$ serial dilutions of samples in $0.9 \%$ sodium chloride on $\mathrm{MH}$ agar plates. A bactericidal effect was defined as $\mathrm{a} \geq 3 \log 10 \mathrm{cfu} / \mathrm{mL}$ decrease compared with the initial inoculum.

\section{Cytotoxicity assay}

Cytotoxicity analysis was performed on the HEK293 human embryonic kidney cell line using the Cell Counting Kit-8 (CCK-8; Dojindo, Tokyo, Japan). The HEK293T cells were seeded into 96-well plates at $1 \times 10^{4}$ cells/well. After incubation for $24 \mathrm{~h}$ at $37^{\circ} \mathrm{C}$ in a humidified atmosphere, the medium was replaced with fresh medium that contained active compound $(1 \mu \mathrm{g} / \mathrm{mL}$ to $128 \mu \mathrm{g} / \mathrm{mL}$, in 2-fold increments). Three replicate wells were set for each treatment. After incubation for another $48 \mathrm{~h}$, cell growth was assayed with CCK-8. The relative absorbance was recorded at $450 \mathrm{~nm}$.

\section{Nucleotide accession number}

The nucleotide sequence of 16S rRNA gene of strain B7 has been deposited in GenBank under the accession number JX282195.

\section{Results}

\section{Identification of strain B7}

The bacteria strain B7 that is active against MRSA ATCC 43300 and $P$. aeruginosa ATCC 27853 was selected for further investigation. Morphologically, strain B7 was characterized to be a rod-shaped, spore-forming, motile, Gram-positive bacterium. Aerobic growth of B7 occurred at a temperature between 20 and $50^{\circ} \mathrm{C}$ and a $\mathrm{pH}$ between 6 and 8. Optimal conditions included a temperature of $30^{\circ} \mathrm{C}$ and a $\mathrm{pH}$ of 7 . The bacteria strain $\mathrm{B} 7$ was negative for urease and positive for catalase, oxidase, methyl red test, and nitrate reduction. Starch, chitin, and gelatin were hydrolyzed by strain B7. Acid was produced from D-mannitol, D-gentiobiose, D-xylose, D-Mannose, L-arabinose, mannitol, and glucose. The $\mathrm{G}+\mathrm{C}$ content of the strain DNA was $54.2 \%$. The major fatty acid of strain B7 was anteiso- $\mathrm{C}_{15: 0}$, making up to $50.12 \%$ of the total fatty acids, a characteristic of the genus Paenibacillus. The B7 isolate and P. ehimensis IFO $15659^{\mathrm{T}}$ showed identical 16S rRNA gene sequences [20], which suggests that they are members of the same species. This inference was further confirmed by the DNA-DNA hybridization results. The DNA-DNA re-association between strain B7 and P. ehimensis IFO $15659^{\mathrm{T}}$ was $96.3 \%$. All of these characteristics supported the identification of the isolate as a member of $P$. ehimensis. Thus, strain B7 was named P. ehimensis B7.

\section{Purification of antibiotics produced by P. ehimensis B7}

P. ehimensis $\mathrm{B} 7$ grew well and produced active compounds in the KL medium. Bioactivity was detectable approximately $20 \mathrm{~h}$ after inoculation and reached a maximum level at $96 \mathrm{~h}$. The cultures were separated into supernatant and cell pellets by centrifugation. Before purification, the stability of the antibiotics that were present in the culture supernatant was investigated according to a previously described method [15]. The active compounds were stable at a $\mathrm{pH}$ of 2.0 to 8.0, and their antimicrobial activities were also not affected by heat treatment at 40 or $80^{\circ} \mathrm{C}$ for $1 \mathrm{~h}$.

The antibiotics were easily absorbed from the culture supernatant by Amberlite XAD-16 resin. The resin was washed with distilled water and then eluted with stepwise gradients of aqueous methanol. One fraction that was eluted with $100 \%$ methanol exhibited the most significant antimicrobial activity. This fraction was extracted with a SPE cartridge and further separated by HPLC. Two active compounds that were eluted at retention times of 28.2 and 26.4 min were obtained and named PE1 and PE2, respectively. The final yield was approximately $17.6 \mathrm{mg} / \mathrm{L}$ for PE1 and $12.3 \mathrm{mg} / \mathrm{L}$ for PE2.

\section{Structure analysis}

ESI-MS analysis indicated that PE1 had a molecular mass of 1114 Da, and PE2 had a molecular weight of 1,100 Da. The two molecular masses differed from each other by 14 $\mathrm{Da}$, suggesting that they were homologues. Amino acid analysis demonstrated that these two compounds had the same amino acid composition, and both of them contained L- 2,4-diaminobutyric acid (L-Dab), L-leucine (L-Leu), L-isoleucine (L-Ile), L-threonine 
(L-Thr), D-Phenylalanine (D-Phe), and D-valine (D-Val), with molar ratios of 3:2:1:1:1:1, which further confirmed that they were structural close-related peptide antibiotics.

After alkaline hydrolysis (1 M KOH, $8 \mathrm{~h}$, room temperature), the purified compounds with protonated molecular masses of 1,115 and 1,101 Da, yielded two new products, with $[\mathrm{M}+\mathrm{H}]^{+}$ions of 1,133 and $1,119 \mathrm{Da}$, respectively. The $18 \mathrm{Da}$ increase in mass was attributed to the hydrolysis of a lactone. This result indicated that the two compounds were cyclic lipopeptide antibiotics. The MS/MS spectrum of the doubly charged precursor ion of the hydrolyzed compound at $\mathrm{m} / \mathrm{z} 567.4$ with a mass of 1133 Da was shown in Figure 1. Successive fragmentations from the two termini of the ring-opened lipopeptide resulted in b-type ions at $\mathrm{m} / \mathrm{z} 1014.3$, 901.2, 802.1, 702.1, 589.1, 441.9, 341.9, and 228.8, along with corresponding $\mathrm{y}$-type ions detected at $\mathrm{m} / \mathrm{z}$ 905.2, 792.1, 692.0, 544.9, 431.9, 331.6, and 232.7. These fragment ions allowed for the assignment of the following sequence: Ile/Leu-Dab-Phe- Leu/Ile-Dab-Val-Leu/IleThr-OH. The b-type ions at $\mathrm{m} / \mathrm{z} 228.8$ corresponded to fatty acid (FA)-Dab, which indicated that the fatty acyl moiety has the elemental composition of $\mathrm{C}_{7} \mathrm{H}_{12} \mathrm{O}_{2}$.

The ring-opened PE2 with a mass $[\mathrm{M}+\mathrm{H}]^{+}$of 1,119 $\mathrm{Da}$ was also analyzed by CID. The tandem mass spectrum of this derivative was shown in Figure 2. All of the b-type ions that were generated from this doubly charged precursor ion $[\mathrm{M}+2 \mathrm{H}]^{2+}$ at $\mathrm{m} / \mathrm{z} 560.3$ were 14. $\mathrm{Da}$ less than those generated from the precursor ion $[\mathrm{M}+2 \mathrm{H}]^{2+}$ at $\mathrm{m} / \mathrm{z}$ 567.4. However, the two $\mathrm{y}$-type ion series for the two compounds were almost the same in mass, which indicated that the two compounds had identical amino acid sequences but different fatty acid chains. Similar to PE1, PE2 also produced a fragment ion at $\mathrm{m} / \mathrm{z}$ 905.1, which corresponded to the loss of 214 Da from the $[\mathrm{M}+\mathrm{H}]^{+}$ion. Examination of the neutral fragment that was lost suggested that it contains a Dab residue and a fatty acyl moiety $\left(\mathrm{C}_{6} \mathrm{H}_{10} \mathrm{O}_{2}\right)$. These results further confirmed that the two compounds were different in their fatty acyl moieties.

Apart from in the C-terminal amino acid (Thr), no hydroxyl group was found in the peptide moieties of $P$. ehimensis lipopeptides studied here. Thus, a lactone linkage was only formed between the carboxyl group of the C-terminal and the hydroxyl group of fatty acid moieties. The proposed structures for PE1 and PE2 are showed in Figure 3.

\section{Antimicrobial activities of the purified compounds}

The antimicrobial activities of the purified compounds PE1 and PE2 were measured using micro dilution methods. Table 1 showed that PE1 and PE2 both had a similar level of strong activity against all of the tested Gram-positive and Gram-negative pathogens as well as Candida albicans. The MICs of the compounds were lower than those of commercial polymyxin B in the cases of Gram-positive bacteria. In the case of $E$. coli ATCC 35318, E. coli 5539, and P. aeruginosa ATCC 27853, the MICs of PE1 and PE2 were higher than that of polymyxin B. Interestingly, P. aeruginosa 5215 , a pandrug resistant clinical isolate, was highly sensitive to PE1 and PE2, with MICs of $2 \mu \mathrm{g} / \mathrm{mL}$ that was slightly lower than that of polymyxin $\mathrm{B}$.

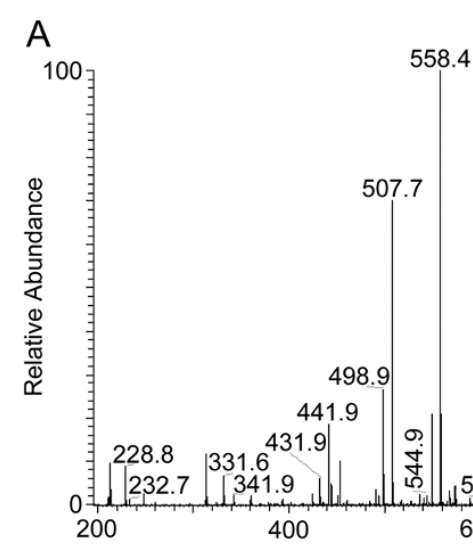

B

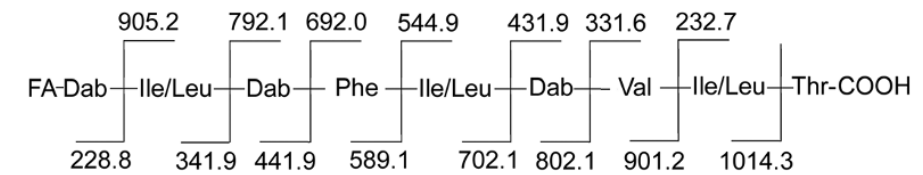

Figure $1 \mathrm{MS} / \mathrm{MS}$ spectrum of PE1 and its proposed amino acid sequence. (A) MS/MS spectrum of the doubly charged precursor ion at $\mathrm{m} / \mathrm{z}$ 567.4 of the hydrolyzed PE1 of 1,133 Da. (B) Proposed amino acid sequence of PE1. 


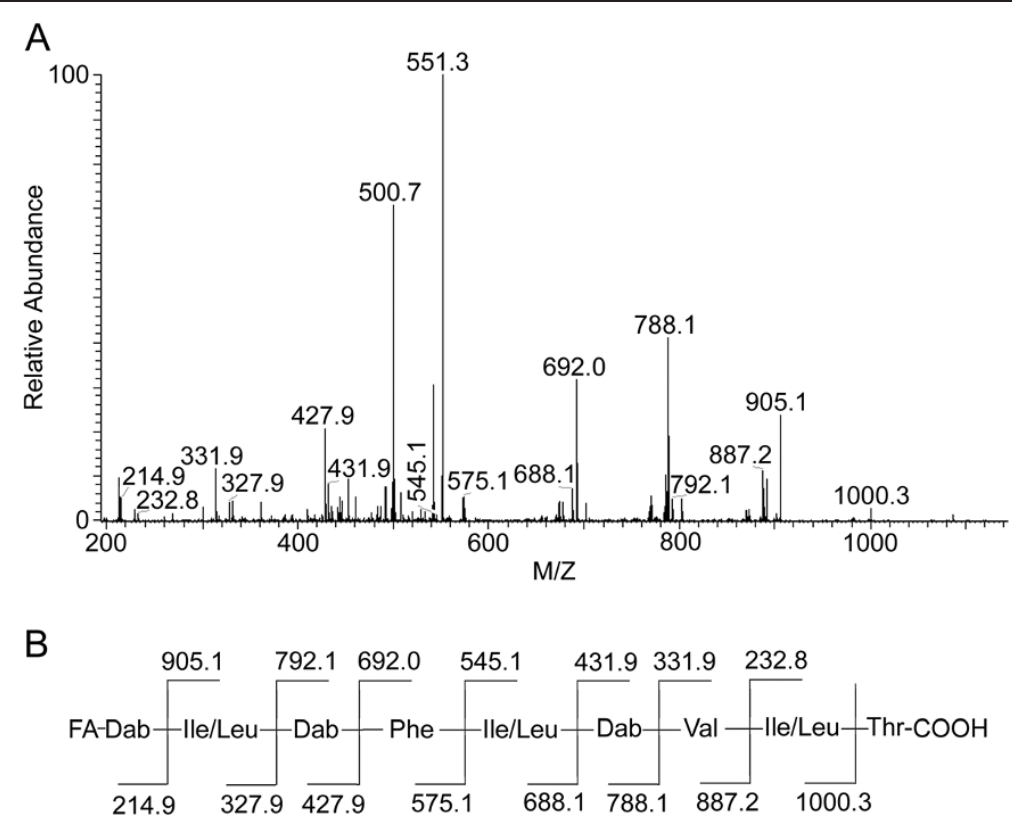

Figure 2 MS/MS spectrum of PE2 and its proposed amino acid sequence. (A) MS/MS spectrum of the doubly charged precursor ion at $\mathrm{m} / \mathrm{z}$ 560.3 of the hydrolyzed PE2 of 1,119 Da. (B) Proposed amino acid sequence of PE2.

\section{Time-kill assays}

To further evaluate the growth inhibition effect of newly isolated antibiotics, killing experiments of PE1 and PE2 against $S$. aureus ATCC 43300 and $P$. aeruginosa ATCC 27853 were performed. The time-kill curves of PE1 against both strains were similar to PE2 (Figure 4). In the case of $P$. aeruginosa ATCC 27853, all of the tested antibiotics at $4 \times$ MIC rapidly reduced the number of viable cells of this strain by at least 3 orders of magnitude over the first $3 \mathrm{~h}$ of exposure, and no bacteria could be detected after a $24 \mathrm{~h}$ incubation. In the case of $S$. aureus ATCC 43300, the number of viable cells counted also dramatically decreased within a period of $3 \mathrm{~h}$ following the addition of these two compounds, although substantial re-growth occurred after $24 \mathrm{~h}$. Thus, PE1 and PE2 were determined to be bactericidal at high concentrations, which is consistent with the characteristics of other cationic cyclic lipopeptides [21,22].

\section{Effect of divalent cations on antibacterial activity}

To determine the effect of divalent cations on the antibacterial activity of the lipopeptides that are produced by $P$. ehimensis B7, the MICs of PE1 against S. aureus ATCC 43300 and $P$. aeruginosa ATCC 27853 were determined in

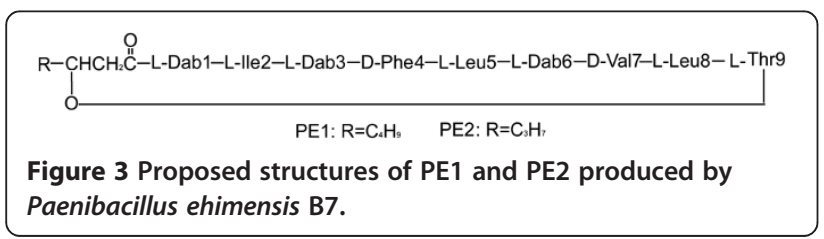

$\mathrm{MH}$ medium with $10 \mathrm{mM} \mathrm{Ca}^{2+}$ or $\mathrm{Mg}^{2+}$. In normal medium, the MICs of PE1 for S. aureus ATCC 43300 and $P$. aeruginosa ATCC 27853 were 4 and $8 \mu \mathrm{g} / \mathrm{mL}$, respectively. However, the MICs of PE1 for $S$. aureus ATCC 43300 and $P$. aeruginosa ATCC 27853 increased to 8 and $>64 \mu \mathrm{g} / \mathrm{mL}$, respectively, when $10 \mathrm{mM} \mathrm{CaCl} \mathrm{mas}_{2}$ wa added to the test medium. Similar results were obtained when $\mathrm{Mg}^{2+}$ was added instead of $\mathrm{Ca}^{2+}$ (Table 2).

\section{Cytotoxicity}

The cytotoxicity of the purified compounds (PE1 and PE2) against mammalian cells was tested by the CCK- 8 assay. PE1 and PE2 showed little cytotoxicity against HEK293T cells (treatment time, $24 \mathrm{~h}$ ) at all of concentrations that were tested $(1 \mu \mathrm{g} / \mathrm{mL}$ to $128 \mu \mathrm{g} / \mathrm{mL}$ ) (Figure 5 ).

Table 1 The minimum inhibitory concentrations (MICs) of lipopeptide antibiotics (PE1 and PE2) produced by Paenibacillus ehimensis B7

\begin{tabular}{lccc}
\hline Indicator strain & \multicolumn{3}{c}{ MIC $(\boldsymbol{\mu g} / \mathbf{m L})$} \\
\cline { 2 - 4 } & PE1 & PE2 & polymyxin B \\
\hline Staphylococcus epidermidis CMCC 26069 & 1 & 1 & 4 \\
Staphylococcus aureus ATCC 25923 & 8 & 8 & 64 \\
Staphylococcus aureus ATCC 43300 & 4 & 4 & 32 \\
Escherichia coli ATCC 35318 & 8 & 8 & 2 \\
Escherichia coli 5539 & 4 & 4 & 1 \\
Pseudomonas aeruginosa ATCC 27853 & 8 & 4 & 2 \\
Pseudomonas aeruginosa 5215 & 2 & 2 & 4 \\
Candida albicans ATCC 10231 & 8 & 8 & 64 \\
\hline
\end{tabular}



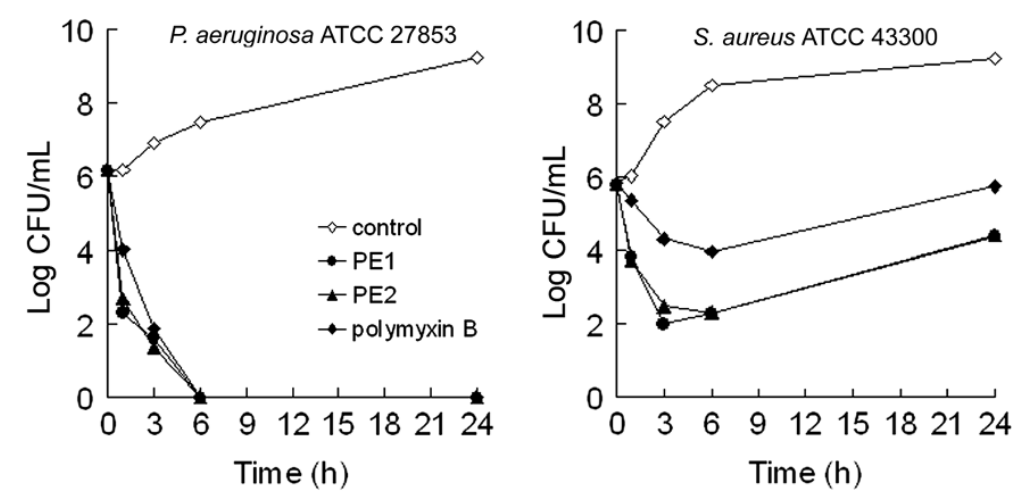

Figure 4 Growth curves of Pseudomonas aeruginosa ATCC 27853 and Staphylococcus aureus ATCC 43300 treated with $4 \times$ MIC peptide antibiotics. The curves are viable cell concentrations plotted against time. In two panels, non-antibiotic control, open diamond; $4 \times$ MIC PE1, filled circle; $4 \times$ MIC PE2, filled triangle; $4 \times$ MIC polymyxin B, filled diamond. For the two strains in the present study, time-kill assays were independently performed 3 times and similar results were obtained. Mean values of the triplicate $\mathrm{cfu} / \mathrm{mL}$ measurements from a single experiment are plotted.

\section{Discussion}

In the present study, B7, a new bacterial strain with potent antimicrobial activity was isolated from a dairy waste sample, and identified as $P$. ehimensis. Phylogenetic analysis based on $16 \mathrm{~S}$ rRNA gene indicated that the isolate was closely related to $P$. elgii, $P$. koreensis, and P. tianmuensis (data not shown). This group of bacteria produces diverse antimicrobial agents, including lipopeptides [15,22,23], lantibiotics [24] and macrolide [14]. Interestingly, most extensively described lipopeptide antibiotics from this group of bacteria contain a high percentage of both Dab and a C6-C7 N-terminal fatty acyl chain [15,22].

The active compounds (PE1 and PE2) that are produced by $P$. ehimensis B7 were structurally similar to the lipopeptide polypeptins (A and B) that were previously isolated from Bacillus circulum [25]. Polypeptin is a group of polypeptide antibiotics composed of a cyclic nonapeptide moiety and a fatty acid side chain. To date, five polypeptin-type antibiotics, including polypeptin A, polypeptin B, permetin A, BMY-28160, and pelgipeptin $D$, have been extensively described [25-28]. Polypeptins $A$ and $B$, which have the same molecular formula, have identical amino acid moieties but vary in the structure of fatty acids. BMY-28160 and permetin A only differ from each other at position 2 in peptide moieties (i.e., L-Val is

Table 2 Effect of divalent cations on antibacterial activity of lipopeptide antibiotics (PE1 and PE2) produced by Paenibacillus ehimensis B7

\begin{tabular}{lcc}
\hline Antibiotic & \multicolumn{2}{c}{ MIC $(\boldsymbol{\mu g} / \mathrm{mL})$} \\
\cline { 2 - 3 } & $\begin{array}{c}\text { P. aeruginosa } \\
\text { ATCC 27853 }\end{array}$ & $\begin{array}{c}\text { S. aureus } \\
\text { ATCC 43300 }\end{array}$ \\
\hline PE1 & 8 & 4 \\
PE1 $+10 \mathrm{mM} \mathrm{CaCl}_{2}$ & $>64$ & 8 \\
PE1 $+10 \mathrm{mM} \mathrm{MgCl}_{2}$ & $>64$ & 8 \\
\hline
\end{tabular}

in BMY-28160, and L-Ile in permetin A). Pelgipeptin D and permetin A only differ from each other in the fatty acid moiety, while permetin A differs from polypeptin A only in the amino acid at position 9 (i.e., L-Ser is present in permetin A, and L-Thr in polypeptin A). Polypeptintype antibiotics were known to have a broad spectrum of antimicrobial activity against many Gram-positive and Gram-negative bacteria [25]. The molecular mass of PE1 was identical to that of polypeptin $A$ and $B$, and the amino acid sequences and antimicrobial spectra were extremely similar, suggesting PE1 and polypeptin (A or B) are most likely the same compound. However, PE2 was characterized as a new analog of polypeptin because PE2 and polypeptin contained identical peptide moieties, but differed from each other by a molecular mass of $14 \mathrm{Da}$

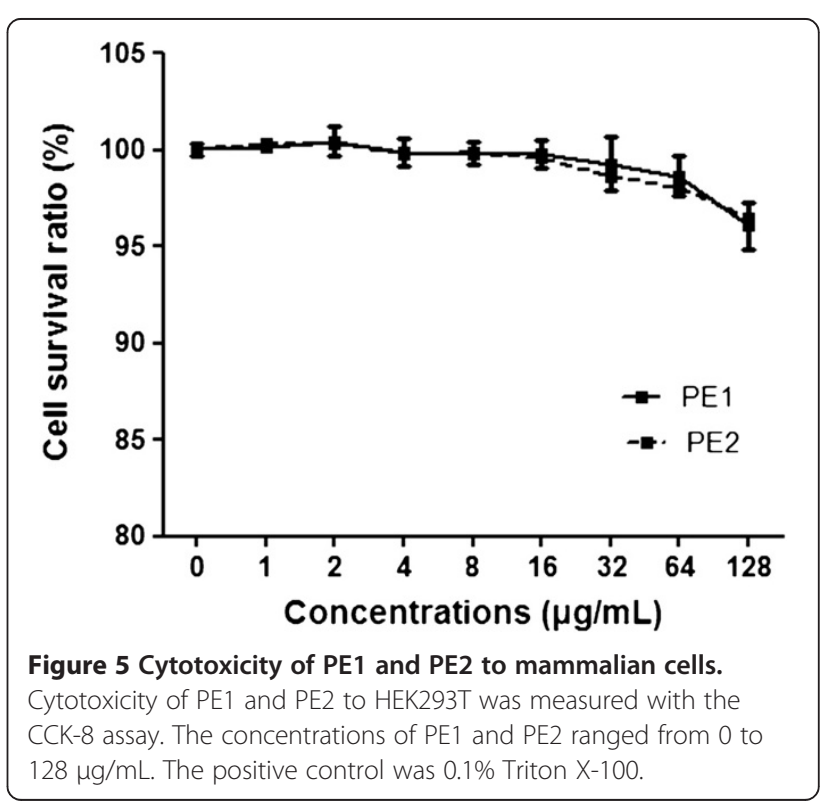


in fatty acid chains. Although Ile and Leu cannot be distinguished by tandem mass spectrometry, there are two reasons for us to assign Ile at position 2, and Leu at positions 5 and 8 in the PE peptide antibiotics (Figure 4). Firstly, the amino acid compositions of $\mathrm{PE}$ and polypeptin are identical, including the molar ratios of amino acids and their absolute configurations. Secondly, positions 5 and 8 of the peptide moieties in all of the extensively described members of polypeptin are Leu, whereas Ile or Val is present in their peptide moieties at position 2 [15,25].

The active modes of cationic lipopeptides generally involve the interaction of positive charged residues with bacterial cell wall, which is normally stabilized by divalent cations $\left(\mathrm{Ca}^{2+}\right.$ and $\left.\mathrm{Mg}^{2+}\right)[8,29,30]$. This is consistent with our results that the addition of $10 \mathrm{mM} \mathrm{Ca}{ }^{2+}$ or $\mathrm{Mg}^{2+}$ significantly reduced the susceptibility of Gram-negative and Gram-positive bacteria to lipopeptides from $P$. ehimensis. In addition to positive residues, the fatty acyl chain and amphipathic structure also contribute to the antimicrobial activity of cationic peptides [12,31]. Although polypeptin and polymyxin are structurally related cyclic lipopeptides with several basic amino acids, their antimicrobial potencies and spectra are significantly different. Polypeptin has a broad-spectrum activity against Gram-positive and Gramnegative bacteria, whereas polymyxin is potently active mainly against Gram-negative bacteria. The selectivity of lipopeptide antibiotics may be attributable to their differential binding affinities to the external and/or cytoplasmic membrane of Gram-negative and Gram-positive bacteria. Understanding the action mode of polypeptin may provide some useful clues toward developing novel lipopeptide antibiotics.

\section{Conclusion}

In conclusion, two active compounds (PE1 and PE2) were obtained from the newly isolated strain $P$. ehimensis. Structural analysis indicated that they were analogs of polypeptin, and PE2 was characterized as a novel analog of polypeptin. These two compounds showed potent activity against Gram-positive and Gram-negative bacterial pathogens, including MRSA and pan-drug resistant $P$. aeruginosa. Although the present results provide some valuable information about the cyclic lipopeptide antibiotics that are produced by Paenibacillus ehimensis, further studies are needed to determine their potential clinical utility.

\section{Competing interests}

The authors declare to have no competing interests.

\section{Authors' contributions}

$\mathrm{ZH}$ was responsible for designing the study and writing the manuscript. $\mathrm{ZH}$ and $\mathrm{YH}$ performed the strain selection and identification experiments. LS and MS carried out the purification and identification of lipopeptide antibiotics. All authors read and approved the final manuscript.

\section{Acknowledgments}

This work was partly supported by grants from National Natural Science Foundation of China (No. 81000867 and 81272299), "Jiangsu Government Scholarship for Overseas Studies", "Medical Key Professionals Program" and "333 Project" of Jiangsu Province.

\section{Author details}

${ }^{1}$ Clinical laboratory, the Fourth Affiliated Hospital of Soochow University, 200 Huihe Road, Wuxi 214062, China. ${ }^{2}$ Clinical laboratory, the Fourth People's Hospital of Wuxi, 200 Huihe Road, Wuxi 214062, China. ${ }^{3}$ Institute for the Control of Agro-chemicals of Zhejiang Province, 29 East Fengqi Road, Hangzhou 310020, China.

Received: 9 October 2012 Accepted: 11 April 2013

Published: 17 April 2013

\section{References}

1. Arias CA, Murray BE: Antibiotic-resistant bugs in the 21st century-a clinical super-challenge. New Engl J Med 2009, 360(5):439-443.

2. Fischbach MA, Walsh CT: Antibiotics for emerging pathogens. Science 2009, 325(5944):1089-1093.

3. Klevens RM, Edwards JR, Richards CL, Horan TC, Gaynes RP, Pollock DA, Cardo DM: Estimating health care-associated infections and deaths in US hospitals, 2002. Public Health Rep 2007, 122(2):160

4. Benquan W, Yingchun T, Kouxing Z, Tiantuo Z, Jiaxing Z, Shuqing T: Staphylococcus heterogeneously resistant to vancomycin in China and antimicrobial activities of imipenem and vancomycin in combination against It. J Clin Microbiol 2002, 40(3):1109-1112.

5. Zhang R, Eggleston K, Rotimi V, Zeckhauser RJ: Antibiotic resistance as a global threat: evidence from China, Kuwait and the United States. Global Health 2006, 2(6):1-14.

6. Peleg AY, Hooper DC: Hospital-acquired infections due to gram-negative bacteria. New Engl J Med 2010, 362 (19):1804-1813.

7. Pagès $J M$, James $C E$, Winterhalter $M$ : The porin and the permeating antibiotic: a selective diffusion barrier in Gram-negative bacteria. Nat Rev Microbiol 2008, 6(12):893-903.

8. Velkov T, Thompson PE, Nation RL, Li J: Structure-Activity Relationships of Polymyxin Antibiotics. J Med Chem 2010, 53(5):1898.

9. Vaara M, Siikanen O, Apajalahti J, Fox J, Frimodt-Møller N, He H, Poudyal A Li J, Nation RL, Vaara T: A novel polymyxin derivative that lacks the fatty acid tail and carries only three positive charges has strong synergism with agents excluded by the intact outer membrane. Antimicrob Agents Chemother 2010, 54(8):3341-3346.

10. Payne DJ, Gwynn MN, Holmes DJ, Pompliano DL: Drugs for bad bugs: confronting the challenges of antibacterial discovery. Nat Rev Drug Discov 2006, 6(1):29-40

11. He Z, Kisla D, Zhang L, Yuan C, Green-Church KB, Yousef AE: Isolation and identification of a Paenibacillus polymyxa strain that coproduces a novel lantibiotic and polymyxin. Appl Environ Microbiol 2007, 73(1):168-178.

12. Guo Y, Huang E, Yuan C, Zhang L, Yousef AE: Isolation of a Paenibacillus sp. Strain and Structural Elucidation of Its Broad-Spectrum Lipopeptide Antibiotic. Appl Environ Microbiol 2012, 78(9):3156-3165.

13. Delves-Broughton J: Nisin and its application as a food preservative. Int $J$ Dairy Technol 2007, 43(3):73-76.

14. Wu XC, Qian CD, Fang HH, Wen YP, Zhou JY, Zhan ZJ, Ding R, Li O, Gao H: Paenimacrolidin, a novel macrolide antibiotic from Paenibacillus sp. F6-B70 active against methicillin-resistant Staphylococcus aureus. Microb Biotechnol 2011, 4(4):491-502.

15. Wu XC, Shen XB, Ding R, Qian CD, Fang HH, Li O: Isolation and partial characterization of antibiotics produced by Paenibacillus elgii B69. FEMS Microbiol Lett 2011, 310(1):32-38.

16. Weisburg WG, Barns SM, Pelletier DA, Lane DJ: $16 \mathrm{~S}$ ribosomal DNA amplification for phylogenetic study. J Bacteriol 1991, 173(2):697-703

17. Tamura K, Dudley J, Nei M, Kumar S: MEGA4: molecular evolutionary genetics analysis (MEGA) software version 4.0. Mol Biol Evol 2007, 24(8):1596-1599.

18. Sopirala MM, Mangino JE, Gebreyes WA, Biller B, Bannerman T, Balada-Llasat $J M$, Pancholi P: Synergy testing by Etest, microdilution checkerboard, and time-kill methods for pan-drug-resistant Acinetobacter baumannii. Antimicrob Agents Chemother 2010, 54(11):4678-4683. 
19. Fujii $K$, Ikai $Y$, Oka H, Suzuki M, Harada K: A nonempirical method using LC/MS for determination of the absolute configuration of constituent amino acids in a peptide: combination of Marfey's method with mass spectrometry and its practical application. Anal Chem 1997, 69(24):5146-5151.

20. Lee JS, Pyun YR, Bae KS: Transfer of Bacillus ehimensis and Bacillus chitinolyticus to the genus Paenibacillus with emended descriptions of Paenibacillus ehimensis comb. nov. and Paenibacillus chitinolyticus comb. nov. Int J Syst Evol Microbiol 2004, 54(3):929-933.

21. Li J, Turnidge J, Milne R, Nation RL, Coulthard K: In Vitro Pharmacodynamic Properties of Colistin and Colistin Methanesulfonate against Pseudomonas aeruginosalsolates from Patients with Cystic Fibrosis. Antimicrob Agents Chemother 2001, 45(3):781-785.

22. Qian CD, Wu XC, Teng Y, Zhao WP, Li O, Fang SG, Huang ZH, Gao HC: Battacin (Octapeptin B5), a New Cyclic Lipopeptide Antibiotic from Paenibacillus tianmuensis Active against Multidrug-Resistant Gram- Negative Bacteria. Antimicrob Agents Chemother 2012, 56(3):1458-1465.

23. Chung YR, Kim CH, Hwang I, Chun J: Paenibacillus koreensis sp. nov., a new species that produces an iturin-like antifungal compound. Int I Syst Evol Microbiol 2000, 50(4):1495-1500.

24. Teng Y, Zhao W, Qian C, Li O, Zhu L, Wu X: Gene cluster analysis for the biosynthesis of elgicins, novel lantibiotics produced by paenibacillus elgii B69. BMC Microbiol 2012, 12(1):45.

25. Sogn JA: Structure of the peptide antibiotic polypeptin. J Med Chem 1976, 19(10):1228-1231.

26. Takeuchi Y, Murai A, Takahara Y, Kainosho M: The structure of permetin A, a new polypeptin type antibiotic produced by Bacillus circulans. I Antibiot 1979, 32(2):121.

27. Sugawara K, Konishi M, Kawaguchi H: BMY-28160, a new peptide antibiotic. J Antibiot 1984, 37(10):1257-1259.

28. Ding R, Wu XC, Qian CD, Teng Y, Li O, Zhan ZJ, Zhao YH: Isolation and identification of lipopeptide antibiotics from Paenibacillus elgii B69 with inhibitory activity against methicillin-resistant Staphylococcus aureus. J Microbiol 2011, 49(6):942-949.

29. Falagas ME, Kasiakou SK, Saravolatz LD: Colistin: the revival of polymyxins for the management of multidrug-resistant gram-negative bacterial infections. Clin Infect Dis 2005, 40(9):1333-1341.

30. Hancock REW: Peptide antibiotics. Lancet 1997, 349(9049):418-422.

31. Jenssen H, Hamill P, Hancock REW: Peptide antimicrobial agents. Clin Microbiol Rev 2006, 19(3):491-511.

doi:10.1186/1471-2180-13-87

Cite this article as: Huang et al:: Isolation and partial characterization of cyclic lipopeptide antibiotics produced by Paenibacillus ehimensis B7. BMC Microbiology 2013 13:87.

\section{Submit your next manuscript to BioMed Central and take full advantage of:}

- Convenient online submission

- Thorough peer review

- No space constraints or color figure charges

- Immediate publication on acceptance

- Inclusion in PubMed, CAS, Scopus and Google Scholar

- Research which is freely available for redistribution 